\title{
Xanthogranulomatous cholecystitis: characteristic CT findings
}

\author{
Ankur Arora • Amar Mukund \\ Published online: 14 December 2011 \\ (C) Indian Society of Gastroenterology 2011
}

A 20-year-old lady presented with right-upper-quadrant pain and a palpable tender gall bladder. Laboratory parameters were normal except for alkaline phosphatase $225 \mathrm{IU} / \mathrm{L}$. Contrastenhanced CT revealed over distended gall bladder (GB) with smooth circumferential wall thickening and preserved pericholecystic fat planes. A thick hypodense band was seen all along GB with a continuous mucosal contrast enhancement (Fig. 1). A preoperative diagnosis of xanthogranulomatous cholecystitis (XGC) was subsequently confirmed on histopathology following open cholecystectomy.

$\mathrm{XGC}$ is an uncommon aggressive form of chronic cholecystitis characterized by intramural accumulation of lipidladen macrophages, believed to be triggered by extravasation of bile into the gall bladder wall from ruptured RokitanskyAschoff sinuses [1]. Although, it is widely accepted that the imaging features of XGC are non-specific, recent studies have reported certain specific findings favoring XGC $[1,2]$. Goshima et al. reported that when at least three of the five CT findings were observed in combination, the sensitivity, specificity, and accuracy for the differentiation of XGC from GB carcinoma were $83 \%, 100 \%$ and $91 \%$, respectively. These include: i) diffuse GB wall thickening; ii) a continuous mucosal contrast enhancement; iii) intramural hypo-attenuating nodules or band; iv) absence of macroscopic hepatic invasion; and v) absence of intra-hepatic bile duct dilatation [1]. The hypodense mural band represents the intramural accumulation of lipid-laden macrophages, while continuous mucosal line suggests an intact mucosa as opposed to the disrupted mucosa of GB carcinoma.

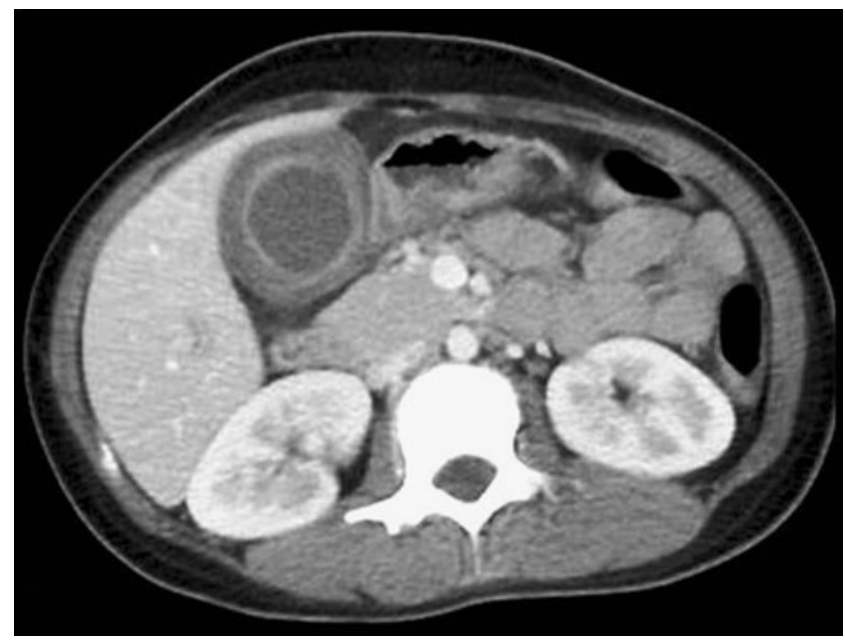

Fig. 1 Contrast-enhanced CT (axial view) reveals smooth circumferential gall bladder wall thickening with a thick hypodense band all along the gall bladder and a continuous contrast - enhanced mucosal line. Pericholecystic fat planes are preserved

\section{References}

1. Goshima S, Chang S, Wang JH, Kanematsu M, Bae KT, Federle MP. Xanthogranulomatous cholecystitis: diagnostic performance of CT to differentiate from gallbladder cancer. Eur J Radiol. 2010;74: e79-83.

2. Uchiyama K, Ozawa S, Ueno M, et al. Xanthogranulomatous cholecystitis: the use of preoperative CT findings to differentiate it from gallbladder carcinoma. J Hepatobiliary Pancreat Surg. 2009;16:333-8.

\footnotetext{
A. Arora $(\bowtie) \cdot$ A. Mukund

Institute of Liver and Biliary Sciences,

New Delhi 110 070, India

e-mail: aroradrankur@yahoo.com
} 